\title{
ARTICLE
}

\section{Functional connectivity of the raphe nucleus as a predictor of the response to selective serotonin reuptake inhibitors in obsessive-compulsive disorder}

\author{
Minah Kim (iD ${ }^{1,2}$, Seoyeon Kwak ${ }^{3}$, Youngwoo Bryan Yoon (iD ${ }^{4}$, Yoo Bin Kwak ${ }^{3}$, Taekwan Kim ${ }^{3}$, Kang Ik K. Cho ${ }^{3}$, Tae Young Lee ${ }^{1}$ and \\ Jun Soo Kwon ${ }^{1,2,3,5}$
}

Selective serotonin reuptake inhibitors (SSRIs) are first-line pharmacological agents for treating obsessive-compulsive disorder (OCD). However, because nearly half of patients show insufficient SSRI responses, serotonergic dysfunction in heterogeneous OCD patients should be investigated for precision medicine. We aimed to determine whether functional connectivity (FC) of the raphe nucleus (RN), the major source of most serotonergic neurons, was altered in OCD patients and could predict the SSRI response. A total of 102 medication-free OCD patients and 101 matched healthy control $(\mathrm{HC})$ subjects participated in resting-state functional magnetic resonance imaging. Among them, 54 OCD patients were treated with SSRIs for 16 weeks, resulting in 26 responders and 28 nonresponders. Seed-based whole brain FC with the RN as a seed region was compared between the OCD and HC groups, as well as between SSRI responders and nonresponders. FC cluster values showing significant group differences were used to investigate factors correlated with symptomatic severity before treatment and predictive of SSRI response. Compared to HCs, OCD patients exhibited significantly larger FC between the RN and temporal cortices including the middle temporal gyrus (MTG), paracingulate gyrus, amygdala, hippocampus, putamen, thalamus, and brain stem. Greater RN-left MTG FC was positively correlated with OC symptom severity at baseline. In addition, larger FC of the RN-left MTG was also found in SSRI nonresponders compared to responders, which was a significant predictor of SSRI response after 16 weeks. The FC of RN may reflect the neurobiological underpinning of $O C D$ and could aid future precision medicine as a differential brain-based biomarker.

Neuropsychopharmacology (2019) 44:2073-2081; https://doi.org/10.1038/s41386-019-0436-2

\section{INTRODUCTION}

Obsessive-compulsive disorder (OCD) is often characterized by intrusive thoughts and repetitive behaviors. Because the current diagnosis of OCD is based on these common symptomatic features, OCD is largely heterogeneous not only in its pathophysiology but also in its treatment response and prognosis [1]. Treatment guidelines recommend selective serotonin reuptake inhibitors (SSRIs) as a first-line medication for moderate-to-severe $\operatorname{OCD}[2,3]$. This treatment has been supported by previous studies showing that serotonergic dysregulation plays a key role in OCD pathophysiology [4-6]. However, $40-60 \%$ of patients do not show sufficient therapeutic responses to SSRIs [7-9], possibly due to heterogeneity in the underlying pathophysiology because of the current symptombased diagnostic standards. Recent evidence suggests the use of dopaminergic or glutamatergic agents in SSRI-nonresponsive OCD patients [10-12]. However, a significant amount of time for trial and error is needed to determine whether a patient is an SSRI responder, which will result in additional suffering for patients. Therefore, predicting SSRI response from the beginning of treatment using brain-based biomarkers would provide valuable information to both psychiatrists and patients in terms of selecting first-line pharmacological agents in precision medicine.

Changes in the brain activity associated with SSRI treatment in OCD have been reported in previous studies using various imaging modalities; such changes include regional brain glucose metabolism [13-15], brain N-acetylaspartate levels [16], functional activity during task performance [17], and the resting-state functional brain connectome [18]. Nevertheless, the prospects for SSRI response prediction using neuroimaging markers have not yet been sufficiently studied. Pretreatment glucose metabolism in the orbitofrontal cortex [15] and brain activation during symptom-provoking tasks [19] are reportedly related to the improvement of obsessive-compulsive (OC) symptom severity after treatment. Shin et al. [18] also showed a strong correlation between changes in the functional brain connectome and improved OC symptoms after SSRI treatment. In addition, Yun et al. [20] demonstrated the use of a machine learning method to classify SSRI responders and nonresponders with high accuracy by brain structural covariance. However, most previous studies have targeted less specific changes in serotonergic activity, which may be a key factor in detecting the serotonin-related pathophysiology of OCD as well as predicting SSRI response.

\footnotetext{
${ }^{1}$ Department of Neuropsychiatry, Seoul National University Hospital, Seoul, Republic of Korea; ${ }^{2}$ Department of Psychiatry, Seoul National University College of Medicine, 101 Daehak-no, Chongno-gu, Seoul 03080, Republic of Korea; ${ }^{3}$ Department of Brain and Cognitive Sciences, Seoul National University College of Natural Science, Seoul, Republic of Korea; ${ }^{4}$ Department of Psychiatry, Washington University in St. Louis, St. Louis, MO, USA and ${ }^{5}$ Institute of Human Behavioral Medicine, SNU-MRC, Seoul, Republic of Korea Correspondence: Jun Soo Kwon (kwonjs@snu.ac.kr)
} 
Serotonergic dysregulation in OCD pathophysiology has mainly been shown in studies using [11C]DASB positron emission tomography (PET) to investigate the serotonin transporter (SERT) binding potential in specific brain regions of interest (ROls). Reduced SERT availability in subcortical and cortical areas comprising the cortico-striato-thalamo-cortical (CSTC) loop in drug-naïve OCD patients has been reported [21-23]. Because these studies targeted specific ROIs in a relatively small number of subjects, the functional connectivity (FC) between brain areas comprising the CSTC circuit could not be investigated. Furthermore, Kim et al. [4] found that the decreased SERT binding potential in medication-free OCD patients did not change after SSRI treatment, suggesting that the SERT binding potential may not be a sensitive predictor of SSRI response in patients with OCD.

In this context, the raphe nucleus (RN), which is the site of most serotonergic neurons and projects to multiple brain regions, can be a potent seed ROI for investigating FC that may be related to serotonergic signaling. Preece et al. [24] showed for the first time that elevated serotonin levels in rat brains evoked changes in the blood oxygen level-dependent (BOLD) signal on functional magnetic resonance imaging (fMRI) and suggested that the fMRI method could be used as a noninvasive tool to study serotonergic function in animal models, with the potential for clinical application in the human brain. In healthy human volunteers, Beliveau et al. [25] reported significant positive FC of the RN with brain regions involved in cognitive and emotion processing, including the anterior cingulate, amygdala, insula, hippocampus, thalamus, basal ganglia, and cerebellum. Furthermore, they found a significant association between the FC of the RN with those brain regions and the regional SERT binding potential measured by [11C]DASB PET, suggesting that FC of the RN as a seed ROI may be useful in estimating the resting-state FC that is supposed to be related to serotonergic activity. Despite such findings, only a few studies have applied this method in clinical populations with depression [26, 27], and to the best of our knowledge, there is no related study involving patients with $O C D$.

In the present study, we used a large sample of OCD patients to investigate whether resting-state $\mathrm{FC}$ with the $\mathrm{RN}$ as a seed $\mathrm{ROI}$ is altered in OCD and can predict SSRI response. It was hypothesized that compared to healthy control $(\mathrm{HC})$ subjects, medication-free OCD patients would show increased FC between the RN and brain areas comprising the CSTC circuit. In addition, we expected that the FC patterns of the RN would differ between SSRI responders and nonresponders and that the FC would predict the response of OCD patients to 16 weeks of SSRI treatment.

\section{MATERIALS AND METHODS}

\section{Participants}

A total of 102 medication-free OCD patients with moderate-tosevere symptoms and 101 age-matched, sex-matched, and handedness-matched $\mathrm{HC}$ subjects were recruited to participate in resting-state fMRI. Forty-one of the OCD patients were drug naïve, and 61 had not taken medication for at least one month before the fMRI scan. All patients with OCD were recruited from the OCD clinic at Seoul National University Hospital (SNUH) and fulfilled the Diagnostic and Statistical Manual of Mental DisordersIV (DSM-IV) criteria for OCD. The HC subjects were recruited via an online advertisement. In patients with OCD, the Yale-Brown Obsessive Compulsive Scale (Y-BOCS) [28] was used to evaluate the severity of OCD symptoms, and all patients showed Y-BOCS scores $>15$ at the time of enrollment. Depressive and anxious symptoms were assessed using the Hamilton Rating Scale for Depression (HAM-D) [29] and the Hamilton Rating Scale for Anxiety (HAM-A) [30], respectively. Symptom ratings, the duration of illness (DOI), and comorbid psychiatric disorders were assessed by certified psychiatrists during a clinical interview. Age of onset was calculated by subtracting the DOI from the age at the time of study participation. The exclusion criteria for both the OCD and HC groups included a lifetime diagnosis of psychotic disorders, substance abuse or dependence (excluding nicotine), neurological disease or significant head trauma, medical illness with cognitive sequelae, and intellectual disability (intelligence quotient [IQ] $<$ 70).

After a baseline assessment including an fMRI scan, patients with OCD were provided with usual treatment including an SSRI and were reassessed for their clinical symptoms after 16 weeks. A total of 72 patients with OCD participated in the 16-week followup clinical assessment. Eighteen patients who were treated with cognitive-behavioral therapy (CBT) or did not receive any form of treatment were excluded from further analysis (i.e., 12 patients received a combination of CBT and SSRIs, 2 patients received CBT only, and 4 patients received no treatment at all). SSRI response was defined as a patient with OCD exhibiting a $\geq 35 \%$ improvement in Y-BOCS scores [10,31]. Among the 54 OCD patients who were treated with SSRIs without CBT, 26 patients met the criteria for responders, and 28 were nonresponders after 16 weeks of follow-up. The use of medication (including SSRIs) during the 16 weeks was ascertained from a thorough review of medical records.

This study was conducted according to the Declaration of Helsinki and was approved by the Institutional Review Board of SNUH. Each subject received a complete description of the study and provided written informed consent before participation. For the minors who participated in this study, informed consent was obtained from both the participants themselves and their caretakers.

\section{Image acquisition and preprocessing}

Functional and structural images were obtained with a 3.0-tesla Trio MRI scanner (Siemens Magnetom Trio, Erlangen, Germany) with a 12-channel head coil. T1-weighted structural images were acquired with the following parameters: echo time (TE) $=1.89 \mathrm{~ms}$; repetition time $(T R)=1670 \mathrm{~ms}$; field of view $(F O V)=250 \mathrm{~mm}$; flip angle $=9^{\circ} ;$ matrix $=256 \times 256$; voxel size $=1.0 \times 0.98 \times 0.98 \mathrm{~mm}^{3}$; and 208 slices. Resting-state $\mathrm{fMRI}$ images were acquired with the following parameters: $\mathrm{TE}=30 \mathrm{~ms} ; \mathrm{TR}=3500 \mathrm{~ms} ; \mathrm{FOV}=240 \mathrm{~mm}$; flip angle $=90^{\circ}$; matrix $=128 \times 128$; voxel size $=1.9 \times 1.9 \times 3.5$ $\mathrm{mm}^{3}$; and 35 slices. Participants were instructed to relax with their eyes closed but not to fall asleep. A questionnaire was administered to the participants after the scan to ensure that they had not fallen asleep. To minimize possible motion artifacts, we stabilized the subjects' heads with head cushions, and we asked the subjects to move as little as possible during image acquisition. Resting-state fMRI images were acquired for 6 min and $58 \mathrm{~s}$. After image acquisition, all acquired images were visually inspected by independent radiologists for any problems that could have occurred during acquisition.

The first 4 resting-state fMRI scans were discarded to allow for magnetic field saturation. The remaining images were processed using the software Statistical Parametric Mapping (SPM) version 12 (Wellcome Department of Cognitive Neurology, London, UK) and the CONN-fMRI FC toolbox (https://www.nitrc.org/projects/ conn) version 17.f [32]. The 112 remaining functional images were realigned and unwarped to correct for head motion, and we confirmed that no subjects exceeded the head motion criteria, i.e., translation $>2 \mathrm{~mm}$ and rotation $>2^{\circ}$ in any direction. After motion correction, each image underwent slice-timing correction. Artifact Detection Tools (ART)-based functional outlier detection and scrubbing (global-signal $Z$-value $=3$, subject mean motion $=0.05$ $\mathrm{mm}$ ) were carried out to minimize the effect of artifacts, including spiking and motion [33]. Then, functional images were coregistered to each participant's respective T1-weighted structural images. The images were segmented into gray matter (GM), white matter (WM) and cerebrospinal fluid (CSF); spatially normalized to Montreal Neurological Institute (MNI) space; and 
Functional connectivity of the raphe nucleus as a predictor of the...

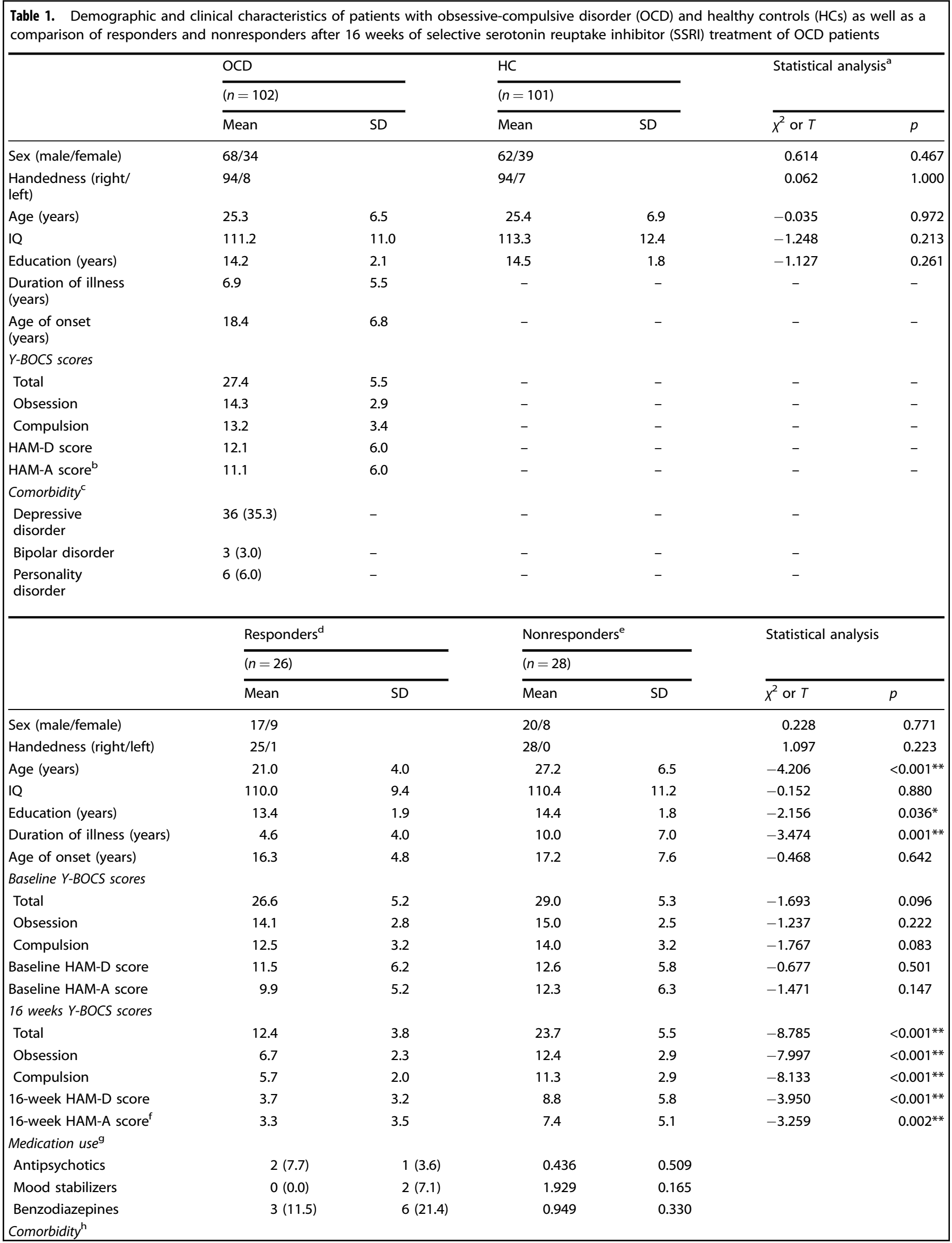


Table 1 continued

\begin{tabular}{|c|c|c|c|c|c|c|}
\hline & \multicolumn{2}{|l|}{ Responders $^{d}$} & \multicolumn{2}{|c|}{ Nonresponders ${ }^{\mathrm{e}}$} & \multirow{2}{*}{\multicolumn{2}{|c|}{ Statistical analysis }} \\
\hline & \multicolumn{2}{|l|}{$(n=26)$} & \multicolumn{2}{|l|}{$(n=28)$} & & \\
\hline & Mean & SD & Mean & SD & $X^{2}$ or $T$ & $p$ \\
\hline None & $17(65.4)$ & $16(57.1)$ & 0.385 & 0.535 & & \\
\hline Depressive disorder & $9(34.6)$ & $8(28.6)$ & 0.228 & 0.633 & & \\
\hline Bipolar disorder & $0(0.0)$ & $3(10.7)$ & 2.950 & 0.086 & & \\
\hline Personality disorder & $0(0.0)$ & $1(3.6)$ & 0.946 & 0.331 & & \\
\hline
\end{tabular}

$S D$ standard deviation, $I Q$ intelligent quotient, $Y$-BOCS Yale-Brown Obsessive-Compulsive Scale; HAM-D Hamilton rating scale for depression, HAM-A Hamilton rating scale for anxiety

*The mean difference is significant at the 0.05 level

**The mean difference is significant at the 0.005 level

andependent $t$-test or Welch's $t$-test if the variances were not equal, $X^{2}$ analysis or Fisher's exact test for categorical data

${ }^{\mathrm{b}}$ The number of missing data was 1

${ }^{\mathrm{c}}$ Number (percentage) of patients who were diagnosed with each comorbid psychiatric disorder; major depressive disorder (MDD) was 6, depressive disorder, not otherwise specified (NOS) was 30, bipolar ii disorder was 2, bipolar disorder, NOS was 1 , schizotypal personality disorder was 3 , obsessive-compulsive disorder was 2, and paranoid personality disorder was 1

${ }^{d}$ Patients with OCD who showed $\geq 35 \%$ reduction in the Y-BOCS total score after 16 weeks of SSRI treatment

ePatients with OCD who showed $<35 \%$ reduction in the Y-BOCS total score after 16 weeks of SSRI treatment

${ }^{\mathrm{f}}$ The number of missing data was 2 in the responder group and 2 in the nonresponder group

${ }^{9}$ Number (percentage) of patients who were prescribed each medication during the 16-week follow-up period

${ }^{\mathrm{h}}$ Number (percentage) of patients who were diagnosed with each comorbid psychiatric disorder; MDD was 3 , depressive disorder, NOS was 5 , bipolar ii disorder was 2, and obsessive-compulsive personality disorder was 1

resampled to $2 \times 2 \times 2 \mathrm{~mm}^{3}$ voxels. A spatial smoothing window with a 4-mm full width at half-maximum (FWHM) isotropic Gaussian kernel was applied. The kernel with a 4-mm FWHM was chosen due to the small size of the RN [34].

The smoothed images underwent nuisance correction with the CompCor method, which regresses out signals from the WM and CSF, motion realignment parameters and their first derivatives. In addition, the CompCor method has been reported to effectively reduce noise from physiological fluctuations [35]. The linear trend was removed throughout the time course, and a temporal bandpass filter of $0.008-0.09 \mathrm{~Hz}$ was subsequently applied.

Functional connectivity analysis

The whole-brain FC patterns were measured with the RN as a seed ROI. The location of the RN was determined from the Harvard Ascending Arousal Network (HAAN) Atlas [36]. Pearson's bivariate correlation analyses were performed to calculate the connection from the RN to other voxels in the whole brain using CONN 17.f. Using a generalized linear model (GLM), the between-group differences in the RN FC patterns were examined by one-way analysis of variance (ANOVA). A false discovery rate (FDR)corrected threshold of $p<0.05$ was applied to report statistically significant clusters.

Statistical analysis

The demographic and clinical characteristics of the participants were compared across groups using independent-samples $t$-tests or, if the variances were not equal, Welch's $t$-tests. The $x^{2}$ test or Fisher's exact test was used to analyze the categorical data. Exploratory Pearson's correlation analysis was performed to assess the relationship between aberrant RN FC found in OCD patients and $\mathrm{OC}$ symptom severity measured by total Y-BOCS scores. To identify factors predictive of the SSRI response, we used binary logistic regression with a backward selection method. A multiple regression analysis with the backward selection method was used to identify the factors that significantly predicted the percent improvement in OC symptom severity during the SSRI treatment period. The independent variables in both the binary logistic regression and the multiple regression analysis included the following: Altered FC of the RN in SSRI nonresponders compared to responders; demographic characteristics that showed differences between the two groups (i.e., age, IQ, years of education, DOI); and baseline scores on the Y-BOCS, HAM-D, and HAM-A.

\section{RESULTS}

Participant characteristics

There was no difference in sex, handedness, age, IQ, or years of education between the OCD and HC groups at baseline. Table 1 summarizes the demographic and clinical characteristics of OCD patients and HCs and of the SSRI responders and nonresponders at baseline and after 16 weeks of treatment. Responders were younger $(t=-4.206, p<0.001)$, less educated $(t=-2.156, p=$ $0.036)$, and had a shorter DOI $(t=-3.474, p=0.001)$ than nonresponders. The severity of OC symptoms, depression, and anxiety did not differ between responders and nonresponders before the start of SSRI treatment. At the time of the 16-week follow-up clinical assessment, escitalopram (10-60 mg per day) was prescribed for 48 patients, fluoxetine (30-80 mg per day) for 9 patients, a combination of escitalopram $(50 \mathrm{mg}$ per day) and fluoxetine (20 mg per day) for 1 patient, and paroxetine $(40 \mathrm{mg}$ per day) for 1 patient. Neither the use of medication other than SSRIs during the 16-week follow-up period nor the presence of comorbid psychiatric disorders assessed at baseline differed between responders and nonresponders. In addition, a comparison of patients with OCD who participated $(n=72)$ and did not participate $(n=30)$ in the follow-up assessment at 16 weeks and a comparison of patients who were included $(n=54)$ and not included $(n=48)$ in the SSRI responder analysis are shown in Tables S1 and S2 in the Supplementary Material.

\section{FC analysis results}

The results of the FC analysis are summarized in Table 2. Compared to $\mathrm{HCs}$, patients with OCD showed greater connectivity between the $\mathrm{RN}$ and various brain regions including the inferior temporal gyri (ITGs) and middle temporal gyri (MTGs) of both hemispheres, the right temporal pole/insular cortex, the paracingulate gyrus, the left amygdala/hippocampus/putamen, right putamen/caudate, thalamus, and brain stem. The FC between the $\mathrm{RN}$ and left occipital pole was smaller in OCD patients than in HCs 
Table 2. Regions where functional connectivity $(\mathrm{FC})$ with the raphe nucleus (RN) was significantly altered in patients with obsessive-compulsive disorder (OCD) compared to healthy controls $(\mathrm{HCs})$ as well as a comparison of responders and nonresponders after 16 weeks of selective serotonin reuptake inhibitor (SSRI) treatment of OCD patients

\begin{tabular}{|c|c|c|c|c|}
\hline Brain region & $\begin{array}{l}\text { MNI coordinate } \\
(x, y, z)\end{array}$ & $\begin{array}{l}\text { Size of clusters } \\
\text { (Number of voxels) }\end{array}$ & \multicolumn{2}{|l|}{ Statistics } \\
\hline \multicolumn{5}{|c|}{ Regions where $F C$ with the $R N$ was larger in $O C D(O C D>H C)$} \\
\hline Left ITG, MTG & $-52,-44,-22$ & 126 & 6.62 & 0.007 \\
\hline Right ITG, MTG & $+60,-42,-10$ & 201 & 5.81 & $<0.001$ \\
\hline Right MTG & $+70,-24,-16$ & 104 & 5.41 & 0.013 \\
\hline Left amygdala, hippocampus, putamen & $-20,-22,-20$ & 155 & 6.02 & 0.003 \\
\hline Right putamen, caudate & $+14,+06,+06$ & 120 & 5.51 & 0.007 \\
\hline Thalamus & $-02,-16,+10$ & 92 & 4.58 & 0.017 \\
\hline Brain stem & $+00,-32,-18$ & 553 & 15.56 & $<0.001$ \\
\hline \multicolumn{5}{|c|}{ Regions where $F C$ with the $R N$ was larger in $H C(H C>O C D)$} \\
\hline \multicolumn{5}{|c|}{ Regions where $F C$ with the $R N$ was larger in SSRI responders (responders ${ }^{b}>$ nonresponders $^{a}$ ) } \\
\hline \multicolumn{5}{|l|}{ None } \\
\hline
\end{tabular}

(Fig. 1). In addition, a comparison of patients with OCD who were included in the SSRI responder analysis $(n=54)$ and those who were not included $(n=48)$ yielded no significant clusters with group differences in the FC analysis.

In the exploratory correlation analysis, the $\mathrm{FC}$ between the RN and left ITG/MTG was significantly related to OC symptom severity, as assessed by total Y-BOCS scores at baseline $(r=$ $0.209, p=0.035$; Fig. S1 in the Supplementary Material). The FC between the RN and the right MTG showed a trend-level correlation with total Y-BOCS scores $(r=0.195, p=0.049)$. A partial correlation analysis controlling for baseline scores on the HAM-D and HAM-A still showed a trend-level correlation between FC of the RN with the left ITG/MTG and OC symptom severity $(r=$ $0.197, p=0.050$ ), whereas the correlation between $\mathrm{FC}$ of the RN with the right MTG and OC symptom severity was not significant $(r=0.175, p=0.084)$.

Patients with OCD who did not respond to 16 weeks of SSRI treatment (i.e., nonresponders) showed significantly larger FC between the RN and the left MTG/superior temporal gyrus (STG) than that of responders (Fig. 2). According to the binary logistic model, smaller FC of the RN with the left MTG/STG and younger age were significant predictors of the response to 16 weeks of SSRI treatment. Multiple regression analysis revealed that smaller FC of the RN with the left MTG/STG and a shorter DOI significantly predicted a subsequent improvement in $\mathrm{OC}$ symptom severity (Table 3; Fig. 2c). The baseline severity of OC, depressive, and anxious symptoms was not included in the significant predictive models produced by regression analyses.

\section{DISCUSSION}

This study is the first to elucidate dysfunctional brain connectivity of the RN, which is a major source of serotonergic neurons, and its usefulness as a predictor of SSRI response in patients with OCD. Medication-free patients with OCD showed greater FC between the $\mathrm{RN}$ and various brain regions comprising the CSTC circuit, including temporal cortices, and larger FC between these areas was correlated with OC symptom severity. Furthermore, OCD patients who did not exhibit a sufficient response to 16 weeks of SSRI treatment showed greater FC between the RN and the left MTG/STG than that of SSRI responders. Furthermore, the altered FC was one of the significant predictors of the SSRI response in patients with OCD when controlling for demographic and clinical characteristics at baseline. Our results not only add to previous literature on the key role of dysfunctional serotonergic activity in OCD pathophysiology but also highlight the possibility of using the FC of the RN as a biomarker of the SSRI response in patients with OCD.

A dysfunctional CSTC circuit in patients with OCD has been consistently shown in previous studies, suggesting that a neurocircuitry approach, updated with recent findings from the temporal cortex and cerebellum applied to the traditional CSTC model, would be a suitable method to elucidate OCD pathophysiology [37-41]. Although previous studies have reported that overactivation of brain areas comprising the CSTC circuit in OCD is decreased after successful treatment [42, 43], network-level alterations of the CSTC circuit have not yet been sufficiently studied in terms of the serotonin-related pathophysiology and treatment response. An animal study conducted by Ahmari et al. [44] revealed that repeated stimulation of the corticostriatal loop produced OCD-like behavior and hyperactivation of striatal neurons in mice, which were reversed by chronic fluoxetine exposure, supporting the hypothesis that serotonin signaling in the CSTC circuit plays an important role in the pathophysiology of OCD. Consistent with that report, the greater RN-left ITG/MTG FC was positively correlated with the symptomatic severity of OCD as measured by the Y-BOCS before treatment in this study. As it has been shown that various brain regions functionally connected to the RN are related to the SERT distribution, which reflects serotonergic activity [25], the results of the current study may suggest altered FC of the RN with brain 


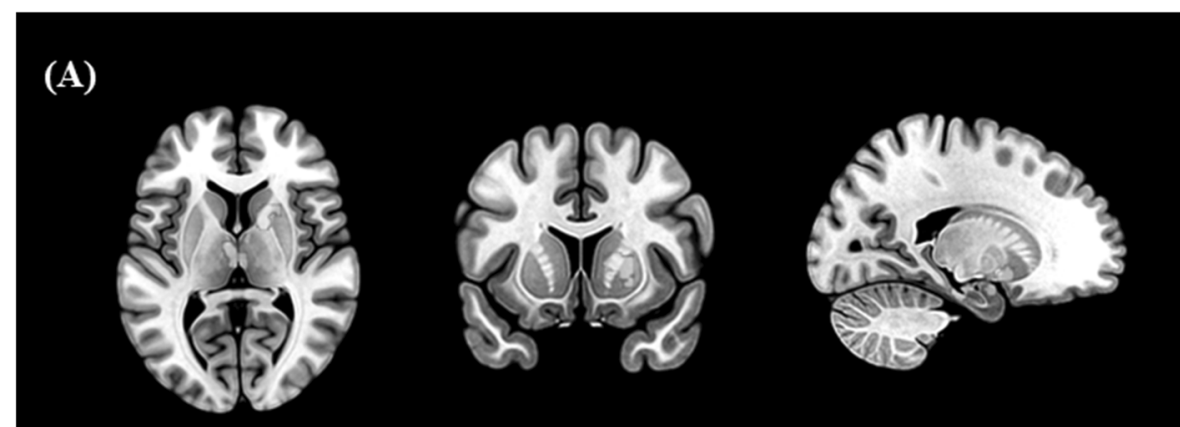

(B)
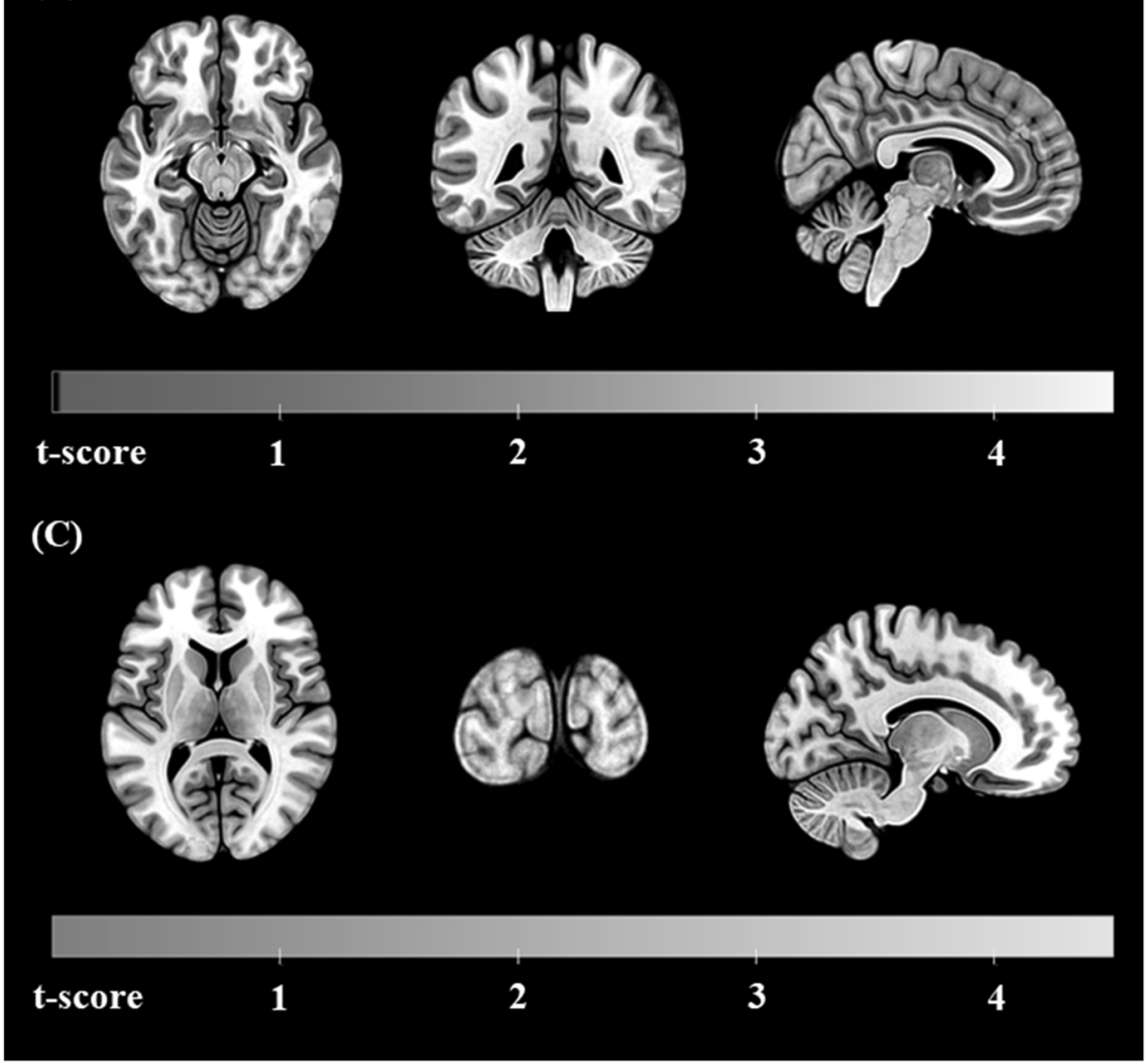

Fig. 1 Statistical maps of the regions where functional connectivity (FC) with the raphe nucleus (RN) was significantly altered in patients with obsessive-compulsive disorder (OCD) compared to healthy controls. The regions where FC with the RN was greater in OCD are marked in redyellow and include the a left amygdala/hippocampus/putamen, right putamen/caudate, thalamus, brain stem, and $\mathbf{b}$ left and right inferior temporal gyri (ITG)/middle temporal gyri (MTGs). The region where FC with the RN was smaller in OCD is marked in blue-green and contains the c left occipital pole. All the results are displayed at a false discovery rate (FDR)-corrected threshold of $p<0.05$. The left hemisphere is depicted on the left in the axial and coronal slices

regions comprising the CSTC circuit as a reflection of the pathophysiological mechanism in patients with OCD.

However, considering that nearly half of patients with OCD show an insufficient therapeutic response to SSRIs and that neurotransmitter systems other than serotonin are also involved in OCD pathophysiology $[8,9,45]$, patients with OCD under the current symptom-based diagnostic standard may be heterogeneous in the degree of serotonergic dysfunction and its contribution to the improvement of OC symptoms. For example, Lee et al. [46] reported that early-onset OCD patients on escitalopram treatment showed less serotonergic pathology, as reflected by the SERT binding potential, than late-onset OCD patients, suggesting that there is significant heterogeneity of serotonergic dysfunction among OCD patients with a symptombased diagnosis. Therefore, investigating brain-based biomarkers to predict SSRI responders would facilitate overcoming those heterogeneities and selecting an appropriate pharmacological agent from the beginning of treatment. We consistently found greater FC between the RN and the left MTG/STG in SSRI nonresponders than in responders, suggesting that altered FC of the RN with the temporal cortices may be a useful brain-based biomarker of the SSRI response. Specifically, in regression models, FC between the RN and left MTG/STG was a significant predictor of the SSRI response, as well as the percent improvement in OC symptom severity after 16 weeks of SSRI treatment, when controlling for the demographic and clinical characteristics measured at baseline. This result is in line with our previous study, which reported individual structural covariance involving the perisylvian area as a predictive factor of SSRI response in patients with OCD [20]. Furthermore, the present results showing 


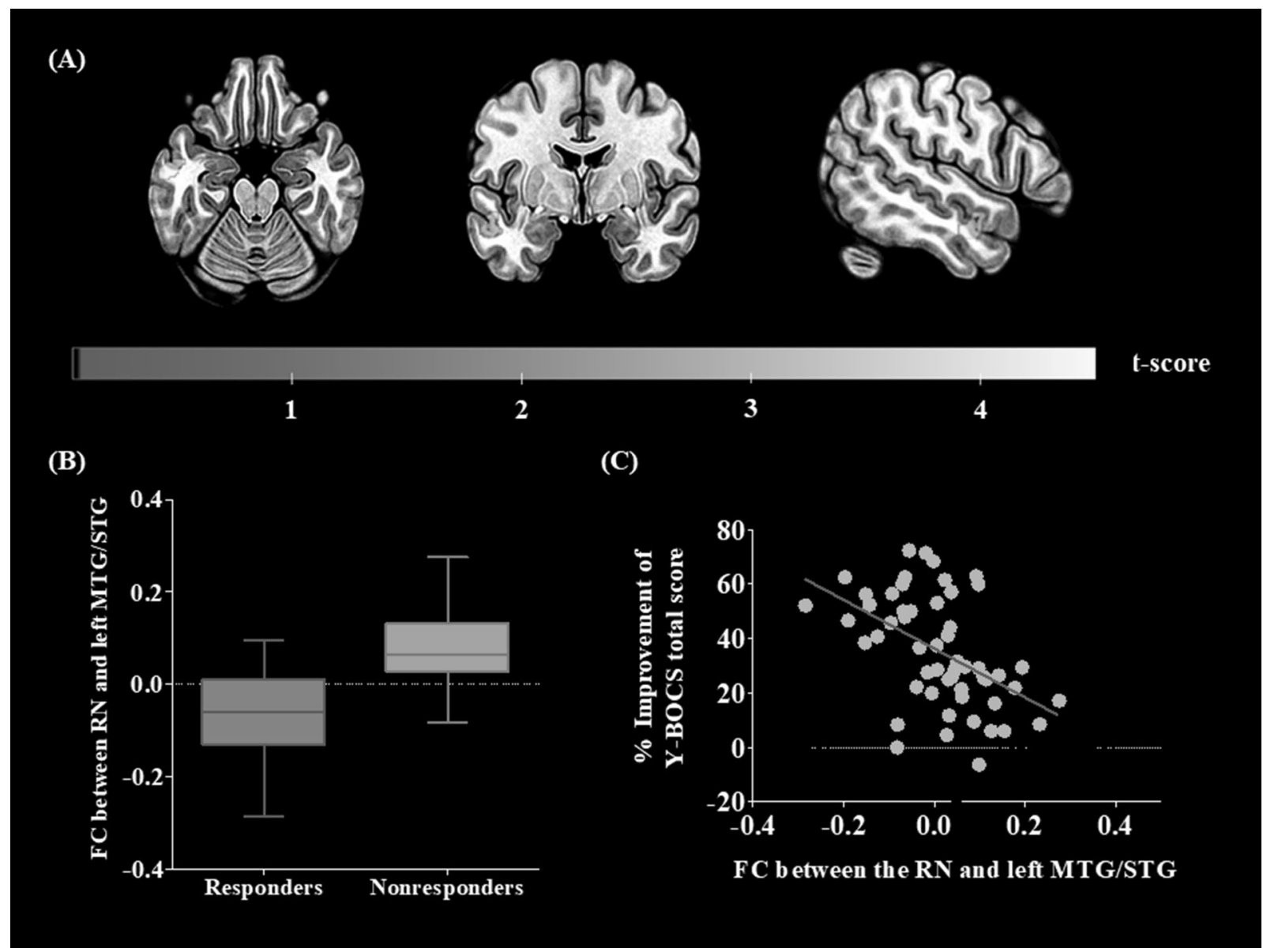

Fig. 2 a Statistical maps of the regions where functional connectivity (FC) with the raphe nucleus (RN) was significantly larger in patients with obsessive-compulsive disorder (OCD) who did not respond to 16 weeks of selective serotonin reuptake inhibitor (SSRI) treatment (nonresponders) than in patients who showed a sufficient response (responders). The region where FC with the RN was larger in SSRI nonresponders is marked in red-yellow and comprises the left middle temporal gyrus (MTG)/superior temporal gyrus (STG). The results are displayed at a false discovery rate (FDR)-corrected threshold of $p<0.05$. The left hemisphere is depicted on the left in the axial and coronal slices. b FC between the RN and the left MTG/STG across SSRI responders and nonresponders. The horizontal lines in the group indicate the means, and the vertical lines in the group indicate the 10th to 90th percentiles. c Correlation between the percent improvement in scores on the Yale-Brown Obsessive Compulsive Scale (Y-BOCS) after 16 weeks of SSRI treatment and FC between the RN and the left MTG/STG at baseline

differential FC of the RN with the left temporal cortex across SSRI responders and nonresponders may indicate a brain-based biomarker of differential serotonergic contributions to OC symptoms under general serotonergic dysfunction shared by heterogeneous OCD groups with a symptom-based diagnosis.

Notably, we found that greater FC between the RN and left temporal cortex not only predicted nonresponse to SSRI treatment but also showed a positive correlation with $\mathrm{OC}$ symptom severity measured before treatment. Therefore, it is pertinent to discuss the importance of the temporal cortex, which is also suggested to be part of the CSTC circuit in recent models [37, 47], in both the pathophysiology of OCD and its response to treatment. Previous studies have reported structural and functional abnormalities of the temporal cortex in OCD patients, suggesting that rich connections of the temporal cortex to OCD-related brain regions may cause those abnormalities $[48,49]$. Furthermore, a recent multicenter volumetric mega-analysis study reported a relative loss of brain volume in the temporal cortices in OCD patients with increasing age in relation to chronic stress and the exacerbated emotional response seen in OCD [47]. The association between temporal cortex pathology and $\mathrm{OC}$ symptoms was also indicated in the high comorbidity of $\mathrm{OC}$ symptoms in patients with temporal lobe epilepsy (TLE) [50].
Specifically, in line with the left lateralization found in the current study, OC symptoms were more prevalent in left-sided TLE patients than in right-sided TLE patients [51]. In addition, Sanematsu et al. [19] showed that pretreatment activation in the left STG during symptom provocation was predictive of improved OC symptoms after treatment using fluvoxamine. Although little is known about the role of the left temporal cortex in the serotonin-related pathophysiology of OCD to date, the results of our study suggest that greater FC between the RN and left temporal cortex may be a candidate biomarker for predicting SSRI response as well as a neurobiological underpinning of OCD.

This study has several limitations. First, although we used the $\mathrm{RN}$ as a seed $\mathrm{ROI}$ in the $\mathrm{FC}$ analysis based on a previous report showing that this FC is correlated with the SERT distribution [25], the $\mathrm{FC}$ of the RN may not exclusively reflect the activity of serotonergic neurons because approximately half of the neurons within the RN are nonserotonergic [52]. The relationship between $\mathrm{FC}$ of the RN and serotonergic signaling is speculative considering the current state of the evidence; thus, the results of this study should be interpreted with caution. Second, the RNs occupy a very small region near the brain stem, which makes them susceptible to motion and physiological noise [53]. Although we had taken 
Table 3. Significant predictors of the treatment response and improvement in Yale-Brown Obsessive-Compulsive Scale (Y-BOCS) scores after 16 weeks of treatment using a selective serotonin reuptake inhibitor (SSRI)

\begin{tabular}{|c|c|c|c|c|c|c|}
\hline Outcome variables & Significant predictors & $R^{2}$ & Exp (B) or Beta & $p$ & \multicolumn{2}{|l|}{$95 \% \mathrm{Cl}$} \\
\hline \multirow[t]{2}{*}{ Response $^{a}$} & FC between the RN and left MTG, STG & 0.460 & 0.000 & $0.002^{* *}$ & 0.000 & 0.003 \\
\hline & Age (years) & & 0.822 & $0.030^{*}$ & 0.688 & 0.981 \\
\hline \multirow[t]{2}{*}{$\%$ Improvement of Y-BOCS total score ${ }^{\mathrm{b}}$} & FC between the RN and left MTG, STG & 0.317 & -68.612 & $0.004^{* *}$ & -114.618 & -22.607 \\
\hline & Duration of illness (years) & & -0.924 & $0.022^{*}$ & -1.772 & -0.147 \\
\hline
\end{tabular}

steps to minimize these effects, including strict exclusion criteria based on motion estimates and the implementation of a tool for correcting physiological noise [25], the remaining physiological noise after those rigorous efforts could have been a potential confounder. Third, we could not acquire the resting-state fMRI data in patients with OCD after finishing 16 weeks of SSRI treatment. The relationship between the symptomatic improvement and the change in FC of the RN to brain areas, the latter of which was suggested as a predictor of the SSRI response in the current study, could be supported by a future study with longitudinal imaging data. Fourth, the participants' resting-state fMRI was acquired with the eyes closed, which might have led to them falling asleep. Although we instructed the participants not to fall asleep and used a questionnaire after the scan to ensure that they had not fallen asleep, the protocol could be stated as a limitation. Finally, the serotonin system is closely related not only to OC symptoms but also to depression and anxiety symptoms. Furthermore, it is known that symptomatic severity and related comorbidities may affect treatment outcomes in addition to brain connectivity [54]. Although the altered FC patterns found in OCD patients were correlated with baseline OC symptom severity and predictive of the SSRI response when baseline depression and anxiety symptoms were controlled as covariates, insufficient consideration of comorbidities, such as not using the Structured Clinical Interview for the Diagnostic and Statistical Manual of Mental Disorders (SCID), remains a limitation of the current study.

Alterations of $\mathrm{FC}$ between the $\mathrm{RN}$ and brain regions comprising the CSTC circuit in medication-free OCD patients highlighted the presence of common serotonergic dysfunctions in OCD pathophysiology. Among those FC changes, greater FC between the RN and left temporal cortex in SSRI nonresponders than in responders may reflect the varying degree and contribution of serotonergic dysfunctions in heterogeneous groups of OCD patients under the current symptom-based diagnostic standards. The results of our study suggest that FC of the RN may be a brain-based biomarker in patients with OCD for future precision medicine.

\section{FUNDING AND DISCLOSURE}

Funding was provided by the Basic Science Research Program and the Basic Research Laboratory Program through the National Research Foundation of Korea (NRF), which is funded by the Ministry of Science, ICT and Future Planning (grant No. 2016R1E1A1A02921618, 2018R1A4A1025891). The authors declare no competing interests.

\section{ADDITIONAL INFORMATION}

Supplementary Information accompanies this paper at (https://doi.org/10.1038/ s41386-019-0436-2)
Publisher's note: Springer Nature remains neutral with regard to jurisdictional claims in published maps and institutional affiliations.

\section{REFERENCES}

1. Lochner C, Stein DJ. Heterogeneity of obsessive-compulsive disorder: a literature review. Harv Rev Psychiatry. 2003;11:113-32.

2. Stein DJ, Koen N, Fineberg N, Fontenelle LF, Matsunaga H, Osser D, et al. A 2012 evidence-based algorithm for the pharmacotherapy for obsessive-compulsive disorder. Curr Psychiatry Rep. 2012;14:211-9.

3. Koran LM, Hanna GL, Hollander E, Nestadt G, Simpson HB, American Psychiatric Association. Practice guideline for the treatment of patients with obsessivecompulsive disorder. Am J Psychiatry. 2007;164:5-53.

4. Kim E, Howes OD, Park JW, Kim SN, Shin SA, Kim BH, et al. Altered serotonin transporter binding potential in patients with obsessive-compulsive disorder under escitalopram treatment: [11C]DASB PET study. Psychol Med. 2016;46:357-66.

5. Hasselbalch SG, Hansen ES, Jakobsen TB, Pinborg LH, Lonborg JH, Bolwig TG. Reduced midbrain-pons serotonin transporter binding in patients with obsessivecompulsive disorder. Acta Psychiatr Scand. 2007;115:388-94.

6. Mundo E, Richter MA, Zai G, Sam F, McBride J, Macciardi F, et al. 5HT1Dbeta receptor gene implicated in the pathogenesis of obsessive-compulsive disorder: further evidence from a family-based association study. Mol Psychiatry. 2002;7:805-9.

7. Bech P, Lonn SL, Overo KF. Relapse prevention and residual symptoms: a closer analysis of placebo-controlled continuation studies with escitalopram in major depressive disorder, generalized anxiety disorder, social anxiety disorder, and obsessive-compulsive disorder. J Clin Psychiatry. 2010;71:121-9.

8. Hirschtritt ME, Bloch MH, Mathews CA. Obsessive-compulsive disorder: advances in diagnosis and treatment. JAMA. 2017;317:1358-67.

9. Kellner M. Drug treatment of obsessive-compulsive disorder. Dialog Clin Neurosci. 2010;12:187-97.

10. Dold M, Aigner M, Lanzenberger R, Kasper S. Antipsychotic augmentation of serotonin reuptake inhibitors in treatment-resistant obsessive-compulsive disorder: an update meta-analysis of double-blind, randomized, placebo-controlled trials. Int J Neuropsychopharmacol. 2015;18:pyv047.

11. Perani D, Garibotto V, Gorini A, Moresco RM, Henin M, Panzacchi A, et al. In vivo PET study of $5 \mathrm{HT}(2 \mathrm{~A})$ serotonin and $\mathrm{D}(2)$ dopamine dysfunction in drug-naive obsessive-compulsive disorder. Neuroimage. 2008;42:306-14.

12. Pittenger $C$. Glutamate modulators in the treatment of obsessive-compulsive disorder. Psychiatr Ann. 2015;45:308-15.

13. Kang DH, Kwon JS, Kim JJ, Youn T, Park HJ, Kim MS, et al. Brain glucose metabolic changes associated with neuropsychological improvements after 4 months of treatment in patients with obsessive-compulsive disorder. Acta Psychiatr Scand. 2003;107:291-7.

14. Perani D, Colombo C, Bressi S, Bonfanti A, Grassi F, Scarone S, et al. [18F]FDG PET study in obsessive-compulsive disorder. A clinical/metabolic correlation study after treatment. Br J Psychiatry. 1995;166:244-50.

15. Saxena S, Brody AL, Maidment KM, Dunkin JJ, Colgan M, Alborzian S, et al. Localized orbitofrontal and subcortical metabolic changes and predictors of response to paroxetine treatment in obsessive-compulsive disorder. Neuropsychopharmacology. 1999;21:683-93.

16. Jang JH, Kwon JS, Jang DP, Moon WJ, Lee JM, Ha TH, et al. A proton MRSI study of brain $\mathrm{N}$-acetylaspartate level after 12 weeks of citalopram treatment in drug-naive patients with obsessive-compulsive disorder. Am J Psychiatry. 2006;163:1202-7. 
17. Nakao T, Nakagawa A, Yoshiura T, Nakatani E, Nabeyama M, Yoshizato C, et al. Brain activation of patients with obsessive-compulsive disorder during neuropsychological and symptom provocation tasks before and after symptom improvement: a functional magnetic resonance imaging study. Biol Psychiatry. 2005;57:901-10

18. Shin DJ, Jung WH, He Y, Wang J, Shim G, Byun MS, et al. The effects of pharmacological treatment on functional brain connectome in obsessive-compulsive disorder. Biol Psychiatry. 2014;75:606-14.

19. Sanematsu H, Nakao T, Yoshiura T, Nabeyama M, Togao O, Tomita M, et al. Predictors of treatment response to fluvoxamine in obsessive-compulsive disorder: an fMRI study. J Psychiatr Res. 2010;44:193-200.

20. Yun JY, Jang JH, Kim SN, Jung WH, Kwon JS. Neural correlates of response to pharmacotherapy in obsessive-compulsive disorder: individualized cortical morphology-based structural covariance. Prog Neuropsychopharmacol Biol Psychiatry. 2015;63:126-33.

21. Matsumoto $R$, Ichise $M$, Ito $H$, Ando $T$, Takahashi $H$, Ikoma $Y$, et al. Reduced serotonin transporter binding in the insular cortex in patients with obsessivecompulsive disorder: a [11C]DASB PET study. Neuroimage. 2010;49:121-6.

22. Hesse $S$, Muller $U$, Lincke $T$, Barthel $H$, Villmann T, Angermeyer MC, et al. Serotonin and dopamine transporter imaging in patients with obsessive-compulsive disorder. Psychiatry Res. 2005;140:63-72.

23. Reimold M, Smolka MN, Zimmer A, Batra A, Knobel A, Solbach C, et al. Reduced availability of serotonin transporters in obsessive-compulsive disorder correlates with symptom severity - a [11C]DASB PET study. J Neural Transm. 2007;114:1603-9.

24. Preece MA, Taylor MJ, Raley J, Blamire A, Sharp T, Sibson NR. Evidence that increased 5-HT release evokes region-specific effects on blood-oxygenation leveldependent functional magnetic resonance imaging responses in the rat brain. Neuroscience. 2009;159:751-9.

25. Beliveau V, Svarer C, Frokjaer VG, Knudsen GM, Greve DN, Fisher PM. Functional connectivity of the dorsal and median raphe nuclei at rest. Neuroimage. 2015;116:187-95.

26. Weinstein JJ, Rogers BP, Taylor WD, Boyd BD, Cowan RL, Shelton KM, et al. Effects of acute tryptophan depletion on raphe functional connectivity in depression. Psychiatry Res. 2015;234:164-71.

27. Ikuta T, Matsuo K, Harada K, Nakashima M, Hobara T, Higuchi N, et al. Disconnectivity between dorsal raphe nucleus and posterior cingulate cortex in later life depression. Front Aging Neurosci. 2017;9:236.

28. Goodman WK, Price LH, Rasmussen SA, Mazure C, Fleischmann RL, Hill CL, et al. The yale-brown obsessive compulsive scale. I. Development, use, and reliability. Arch Gen Psychiatry. 1989;46:1006-11.

29. Hamilton M. A rating scale for depression. J Neurol Neurosurg Psychiatry. 1960;23:56-62.

30. Hamilton $\mathrm{M}$. The assessment of anxiety states by rating. $\mathrm{Br} \mathrm{J}$ Med Psychol 1959;32:50-5.

31. Bloch MH, Bartley CA, Zipperer L, Jakubovski E, Landeros-Weisenberger A, Pittenger $C$, et al. Meta-analysis: hoarding symptoms associated with poor treatment outcome in obsessive-compulsive disorder. Mol Psychiatry. 2014;19:1025-30.

32. Whitfield-Gabrieli S, Nieto-Castanon A. Conn: a functional connectivity toolbox for correlated and anticorrelated brain networks. Brain Connect. 2012; 2:125-41.

33. Power JD, Barnes KA, Snyder AZ, Schlaggar BL, Petersen SE. Spurious but systematic correlations in functional connectivity MRI networks arise from subject motion. Neuroimage. 2012;59:2142-54.

34. Wagner G, Krause-Utz A, De La Cruz F, Schumann A, Schmahl C, Bar KJ. Restingstate functional connectivity of neurotransmitter producing sites in female patients with borderline personality disorder. Prog Neuropsychopharmacol Biol Psychiatry. 2018;83:118-26.
35. Behzadi Y, Restom K, Liau J, Liu TT. A component based noise correction method (CompCor) for BOLD and perfusion based fMRI. Neuroimage. 2007;37:90-101.

36. Edlow BL, Takahashi E, Wu O, Benner T, Dai G, Bu L, et al. Neuroanatomic connectivity of the human ascending arousal system critical to consciousness and its disorders. J Neuropathol Exp Neurol. 2012;71:531-46.

37. Kwon JS, Jang JH, Choi JS, Kang DH. Neuroimaging in obsessive-compulsive disorder. Expert Rev Neurother. 2009;9:255-69.

38. Gu BM, Park JY, Kang DH, Lee SJ, Yoo SY, Jo HJ, et al. Neural correlates of cognitive inflexibility during task-switching in obsessive-compulsive disorder. Brain. 2008;131:155-64.

39. Kim SG, Jung WH, Kim SN, Jang JH, Kwon JS. Disparity between dorsal and ventral networks in patients with obsessive-compulsive disorder: evidence revealed by graph theoretical analysis based on cortical thickness from MRI. Front Hum Neurosci. 2013;7:302.

40. Saxena S, Rauch SL. Functional neuroimaging and the neuroanatomy of obsessive-compulsive disorder. Psychiatr Clin N Am. 2000;23:563-86.

41. Goodman WK, Grice DE, Lapidus KA, Coffey BJ. Obsessive-compulsive disorder. Psychiatr Clin N Am. 2014;37:257-67.

42. Van Der Straten AL, Denys D, Van Wingen GA. Impact of treatment on resting cerebral blood flow and metabolism in obsessive compulsive disorder: a metaanalysis. Sci Rep. 2017;7:17464.

43. Beucke JC, Sepulcre J, Talukdar T, Linnman C, Zschenderlein K, Endrass T, et al. Abnormally high degree connectivity of the orbitofrontal cortex in obsessivecompulsive disorder. JAMA Psychiatry. 2013;70:619-29.

44. Ahmari SE, Spellman T, Douglass NL, Kheirbek MA, Simpson HB, Deisseroth $\mathrm{K}_{t}$ et al. Repeated cortico-striatal stimulation generates persistent OCD-like behavior. Science. 2013;340:1234-9.

45. Pauls DL, Abramovitch A, Rauch SL, Geller DA. Obsessive-compulsive disorder: an integrative genetic and neurobiological perspective. Nat Rev Neurosci. 2014;15:410-24.

46. Lee J, Kim BH, Kim E, Howes OD, Cho KIK, Yoon YB, et al. Higher serotonin transporter availability in early-onset obsessive-compulsive disorder patients undergoing escitalopram treatment: a [(11) C]DASB PET study. Hum Psychopharmacol. 2018;33:e2642.

47. de Wit SJ, Alonso P, Schweren L, Mataix-Cols D, Lochner C, Menchon JM, et al. Multicenter voxel-based morphometry mega-analysis of structural brain scans in obsessive-compulsive disorder. Am J Psychiatry. 2014;171:340-9.

48. Choi JS, Kim HS, Yoo SY, Ha TH, Chang JH, Kim YY, et al. Morphometric alterations of anterior superior temporal cortex in obsessive-compulsive disorder. Depress Anxiety. 2006;23:290-6.

49. Rotge JY, Guehl D, Dilharreguy B, Cuny E, Tignol J, Bioulac B, et al. Provocation of obsessive-compulsive symptoms: a quantitative voxel-based meta-analysis of functional neuroimaging studies. J Psychiatry Neurosci. 2008;33:405-12.

50. Isaacs KL, Philbeck JW, Barr WB, Devinsky O, Alper K. Obsessive-compulsive symptoms in patients with temporal lobe epilepsy. Epilepsy Behav. 2004;5:569-74.

51. Ertekin BA, Kulaksizoglu IB, Ertekin E, Gurses C, Bebek N, Gokyigit A, et al. A comparative study of obsessive-compulsive disorder and other psychiatric comorbidities in patients with temporal lobe epilepsy and idiopathic generalized epilepsy. Epilepsy Behav. 2009;14:634-9.

52. Jacobs BL, Azmitia EC. Structure and function of the brain serotonin system. Physiol Rev. 1992;72:165-229.

53. Brooks JC, Faull OK, Pattinson KT, Jenkinson M. Physiological noise in brainstem FMRI. Front Hum Neurosci. 2013;7:623.

54. Knopp J, Knowles S, Bee P, Lovell K, Bower P. A systematic review of predictors and moderators of response to psychological therapies in OCD: do we have enough empirical evidence to target treatment? Clin Psychol Rev. 2013;33:1067-81. 